\title{
Twinkle Megane: Near-Eye LED Indicators on Glasses for Simple and Smart Navigation in Daily Life
}

\author{
Aryan Firouzian $^{1}$, Yukitoshi Kashimoto ${ }^{2}$, Zeeshan Asghar ${ }^{1,2}$, \\ Niina Keranen ${ }^{1}$, Goshiro Yamamoto ${ }^{2}$, and Petri Pulli ${ }^{1}$ \\ 1 University of Oulu, Box 3000, FI-90014, Finland \\ \{aryan.firouzian, zeeshan.asghar, niina.s.keranen, petri.pulli\}@oulu.fi \\ 2 Nara Institute of Science and Technology, 8916-5, Takayama, Ikoma, Nara Japan \\ \{kashimoto.yukitoshi.km3,goshiro\}@is.naist.jp,
}

\begin{abstract}
We present an eyeglass-type wearable device that has light emitting diode (LED) indicators on the frame of it. The device produces lighting patterns of 14 RGB LEDs near user's eyes as guiding information. Since installed LEDs on the frame of glasses are light and saving power, it is feasible to develop it for daily use. On the other hand, it cannot provide rich information such as text or images. In this study, we aim to realize a remote assistive system that provides assistive commands by lighting patterns of the eyeglass-type device from remote sites. Especially, we consider elderlies who are suffering from mild cognitive impairment as users. They would be one of potential user groups since the device does not block their sights by text or images and it can be worn in daily life without the additional sense of restraint. This paper explains our conceptual assistive system structure, a prototype eyeglass-type device with near-eye LED indicators and usability experimentation in simple navigational tasks.
\end{abstract}

Key words: eyeglass-type wearable device, LED indicators, assistive system, elderly, mild cognitive impairment

\section{Introduction}

Aged society is one of the common crucial issues in the world. In this study, we mainly focus on elderly-user-friendly interface in a telepresence caretaking system. Bharucha et al.[1] estimated 28 million people suffered from dementia in 2009, and it costs 156 billion dollars annually for caretaking them directly. The analysis of the elderly adults demographic suffering from memory loss has drawn attention to the use of technologies, to involve less human and financial resources in the caretaking process. Symptoms of memory problems are categorized into several stages. In most of cases, elderly in an early stage is shifted to a severe stage because of an aggravation of their condition. Considering the increasing population of elderly suffering from memory problems, this study aims to restrain 
the aggravation by supporting elderly in an early stage of memory problem such as mild cognitive impairment.

In the last decades, wearable technologies have been developed to support human activities. Recently, wearable devices can have cameras, display, and some of the sensors on itself. One of the most significant aspects of development is light weight design since it needs to be comfortable for wearing in daily life. In this paper, we show near-eye LED indicators on the frame of glasses as a lightweight wearable device for an assistive interface. Although most of the elderlies suffering mild cognitive impairment can live by themselves, it is necessary to support them in different cases to avoid the aggravation of their condition due to anxiety, etc.

\section{Related Work}

One of the assistive applications for a daily tasks is navigation. In wearable computing research field, navigation has been studied as a major topic. Thomas et al. have developed a hands-free navigational aid by implementing head-mounted display system for outdoor guiding and they believe wearable technology has the potential for wayfinding application [9]. In recent, there are many advanced head-mounted displays such as Google Glass or Epson Moverio BT-200 and so forth. In the future, it is expected that light wearable displays provide highquality images in front of the user. Poppinga et al used off-the-shelf ambiglasses to provide users with notification with LED indicators and intuitive navigation instruction. Our near-eye LED indicator prototype is similar to ambiglasses and it can be customized and combined with advanced head-mounted and give simple navigational instruction. In addition, LED indicators do not disturb a user to see objects located at the center of the user's sight. [8]

\section{Design of Assistive System with Eyeglass-type Device}

We have developed the prototype of the indicator-based smart glasses to provide users with the visual cues, which do not interfere with their vision on the physical environment. Android applications are developed to form messages and send them as binary data to the prototypes. The application has been improved iteratively. The communication protocol between the application and device is promoted to Bluetooth low energy to provide faster communication and consume less power. The first prototype includes 12 LED indicators, while the second prototype has 14 indicators to widen different form of blinking patterns and more meaningful messages. After conducting experimentation phase, test subjects complain about physical design and weight of the device, and it derived us to design more fashionable and light-weight prototype. Figure 1(b) shows the first prototype. Figure 1(a) shows the second prototype, and the position of indicators. We followed similar approach to poppinga et al to form four main navigation commands. However, since our prototype cover more parts of the frame, 
we achieved different results. In addition to LED indicators, the conceptual prototype includes the mounted camera, global positioning system, gyroscope, and step detector sensors. The abovementioned sensors and cameras help the remote caretaker to localize the senior citizens in home and city environment, while the glasses provide the visual cues to guide the users. [2]

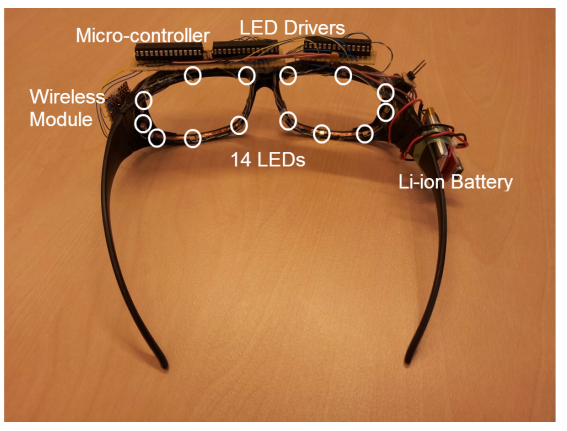

(a)

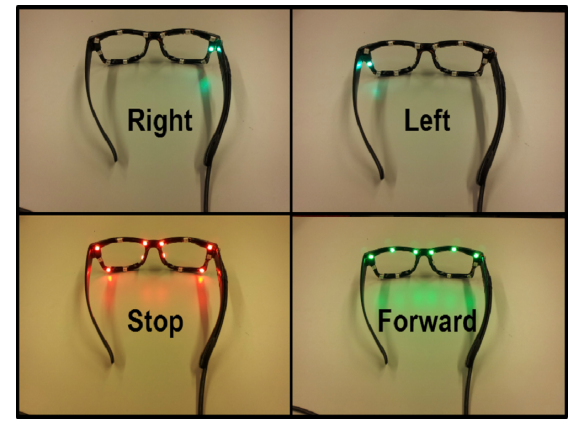

(b)

Fig. 1: (a): Second constructed prototype; white circles depict the position of LED indicators implanted on the frame of the eyeglasses. (b): Lightweight and user friendly design of the prototype

\section{Experimentation of of Near-Eye LED Indicators}

The design science research method is used in the study, and the experiment phase evaluates the usability of the device for further improvement [4]. We designed experiments with different user groups. The Wizard of $\mathrm{Oz}$ method with unstructured interviews, living lab approach, and video recording are used to collect and interpret the data [6].

We previously published a paper to explain settings, protocols and different steps of experimentation with near eye indicator display. The first step is to measure visibility of visual indicators; then forming meaningful notifications with different combination of indicators, and finally evaluate the efficiency of the notification in real life tasks. We conducted controlled experiments to measure satisfactory LED frequencies, brightnesses [7].

In the first experiment, Bayesian analysis indicates what indicators on the frame might be missed when they are blinking, and what indicators might be mistakenly chosen as blinking. In addition, the results clarify the satisfactory blinking pattern for blinking indicators including optimized brightness and frequency. In the second experiment, different sets of LED indicators' combinations blink simultaneously, and users choose interpreted navigational command such as left, right, stop and so on. We found optimized frequency, optimized brightness, 
and most distinguishable indicators on the frame. We formed most meaningful indicators configurations to convey navigational commands. Figure 1(b) shows the four most simple and intuitive navigational commands.

The last experiment evaluates the usability of the visual cues in a navigational task, which is a real life problem for elderly suffering from dementia. We simulate simple navigational tasks for the subjects in an open indoor environment. The users are supposed to walk on predefined map while the visual cues guide them. The map contains a specific number of stops and turning left and right with different rotation degrees. Figure 3 shows the predefined map. One of the main objectives of experiment was to evaluate the simple guidance system in 90-degree and 180-degree turning point.

Two main user groups participate in the experiments and Figure 2 demonstrate the order of the conducted experiments. Eleven student subjects participate in the pilot tests with the average age of 26.05 and the range of 20 to 33 . The results of the Bayesian analysis for the localizing indicators test unveil lowest sensitivity and specificity for nasal indicators (indicators close to the nose). In other word, the nasal LED indicators can be missed while blinking, and they can be mistakenly considered as blinking while they are not. In the next experiment, four out of 48 combinations are chosen as the navigational commands based on the users' preferences. In the last experiment, the student users accomplish their task by following the navigation guidance to walk the predefined route.

\begin{tabular}{|c|c|c|c|}
\hline Localizing Indicators & Indicators Configurations & Walking Navigation & Walking Navigation \\
\hline $\begin{array}{c}\text { First Test: } \\
\text { 3 Student Subjects }\end{array}$ & $\begin{array}{c}\text { Second Test: } \\
\text { 5 Student Subjects }\end{array}$ & $\begin{array}{c}\text { Third Test: } \\
\text { 3 Student Subjects }\end{array}$ & Elderly Subjects \\
\hline
\end{tabular}

Fig. 2: It shows the sequence of the experiments and the number and type of subjects in the experiments.

Eight elderly subjects participate in the usability experiments. Four subjects suffered from severe dementia and four subjects suffered from mild dementia [3]. The severity of dementia deteriorates following track of topic and concentrating conditions [5]. These difficulties prevent severe demented elderly subjects to accomplish their tasks in experiments. Before conducting walking test, we request all the subjects to identify individual blinking indicators and confirm the meaning of navigational cues. The subjects with severe dementia fail in preliminary test and withdraw the experiment.

All the subjects suffering from mild dementia accomplish their tasks by following the visual cues and walking on the predefined route. Table 1 shows the most important collected data in the experiment. The subjects react more accurately to the visual cues at the end part of the walking task. It shows high adaptability to the system in performing the tasks. Figure 3 depicts the map of the navigation area in an open indoor environment. Considering turn-by-turn navigation, the result of the experiment proves that conveying different degree 


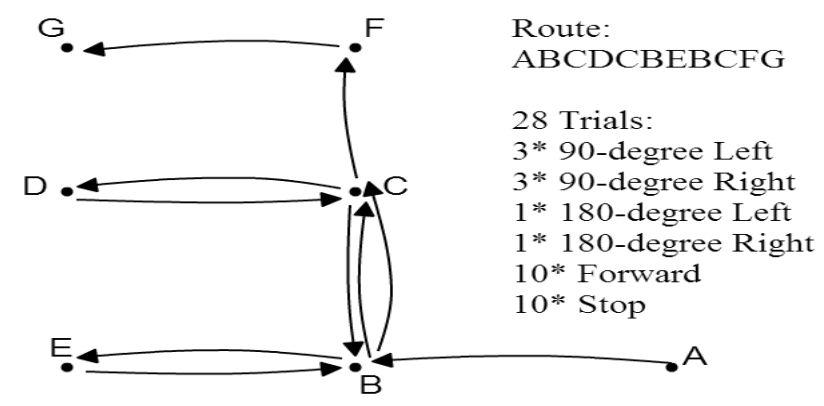

Fig. 3: The predefined map contains number of rotations to the left and right in an open area.

\begin{tabular}{|l||c|c|c|c|}
\hline Variable & Subject A & Subject B & Subject C & Subject D \\
\hline Age & 81 & 80 & 83 & 74 \\
\hline Gender & Male & Male & Female & Male \\
\hline Using eyeglasses & No & Yes & Yes & Yes \\
\hline Mistakes by subjects & 8 & 7 & 7 & 5 \\
\hline Correction via device & 6 & 0 & 3 & 4 \\
\hline Correction via human assistant & 2 & 7 & 4 & 1 \\
\hline Accomplishment time & $220 \mathrm{~s}$ & $390 \mathrm{~s}$ & $286 \mathrm{~s}$ & $136 \mathrm{~s}$ \\
\hline Average speed & $0.068 \mathrm{~m} / \mathrm{s}$ & $0.038 \mathrm{~m} / \mathrm{s}$ & $0.052 \mathrm{~m} / \mathrm{s}$ & $0.110 \mathrm{~m} / \mathrm{s}$ \\
\hline Path length & $15 \mathrm{~m}$ & $15 \mathrm{~m}$ & $15 \mathrm{~m}$ & $15 \mathrm{~m}$ \\
\hline
\end{tabular}

Table 1: The relevant descriptive statistics and factors are measured for four elderly subjects suffering from mild dementia in walking experiment.

angles rotation with simple navigation instruction is the most challenging part. In turning points, subjects can detect the direction that the glasses indicate while the amount of rotation confuse them. The perception of turning commands differ in the subjects, and it means that some subjects always consider turning command as a 90-degree turn and some subjects turn continuously until the stop command is triggered. The indicators blink with the frequency of $1 \mathrm{~Hz}, 1,5 \mathrm{~Hz}$, and $2 \mathrm{~Hz}$ in the pilot and usability experiments. The subjects achieve the best results while indicators blink with the frequency of $1 \mathrm{~Hz}$. Nevertheless, the unstructured interviews uncover that the elderly adults would rather the frequency less than $1 \mathrm{~Hz}$. The test subjects also highly emphasize on the common-shape design of the glasses and they prefer the visual cues not to be seen by outsider to preserve their dignity in real life.

\section{Conclusions}

We have implemented an interface as eyeglasses which are common among senior citizens. The device has the potential to be used in diverse scenarios by 
different target user groups. Most of the users suffering from cognitive decline fail to interact with advanced user interfaces, while our prototype can be used to generate simple notifications for them. There are some tasks such as driving and biking which should not be interrupted, and meanwhile, the user needs to receive information in the attention background. We proposed the mentioned prototype to provide users with simple notifications without distracting them from main tasks. Our future plan includes the evaluation of colors' combinations, indicators' positions, light intensity and blinking pattern to form meaningful notification in different real life challenges and scenarios for both elderly and young users.

\section{Acknowledgment}

We thank staffs in residential nursing care centers of Karpalokoti and ODL for the collaboration in the field testing. We also thank Jarmo Lehtonen and the design team from the University of Lapland for designing conceptual models.

\section{References}

1. A. J. Bharucha, V. Anand, J. Forlizzi, M. A. Dew, C. F. Reynolds, S. Stevens, and H. Wactlar. Intelligent assistive technology applications to dementia care: current capabilities, limitations, and future challenges. The American journal of geriatric psychiatry, 17(2):88-104, 2009.

2. A. Firouzian, Z. Asghar, J. Tervonen, P. Pulli, and G. Yamamoto. Conceptual design and implementation of indicator-based smart glasses: A navigational device for remote assistance of senior citizens suffering from memory loss. In Medical Information and Communication Technology (ISMICT), 2015 9th International Symposium on, pages 153-156. IEEE, 2015.

3. M. F. Folstein, S. E. Folstein, and P. R. McHugh. mini-mental state: a practical method for grading the cognitive state of patients for the clinician. Journal of psychiatric research, 12(3):189-198, 1975.

4. A. Hevner and S. Chatterjee. Design research in information systems: theory and practice, volume 22. Springer Science \& Business Media, 2010.

5. G. Hubbard, M. G. Downs, and S. Tester. Including older people with dementia in research: challenges and strategies. Aging \& Mental Health, 7(5):351-362, 2003.

6. M. Lkk, A. Firouzian, J. Tervonen, G. Yamamoto, and P. Pulli. Attention Control and Eyesight Focus for Senior Citizens, pages 309-315. Virtual, Augmented and Mixed Reality. Applications of Virtual and Augmented Reality. Springer, 2014.

7. S. Manel, H. C. Williams, and S. J. Ormerod. Evaluating presenceabsence models in ecology: the need to account for prevalence. Journal of Applied Ecology, 38(5):921931, 2001.

8. B. Poppinga, N. Henze, J. Fortmann, W. Heuten, and S. Boll. Ambiglassesinformation in the periphery of the visual field. In Mensch $\mathscr{E}$ Computer, pages 153-162, 2012.

9. B. Thomas, V. Demczuk, W. Piekarski, D. Hepworth, and B. Gunther. A wearable computer system with augmented reality to support terrestrial navigation. In Wearable Computers, 1998. Digest of Papers. Second International Symposium on, pages 168-171. IEEE, 1998. 